\title{
Modeling and Simulation of Hydropower Station Diversion System's characteristic line method by introducing water head to flow feedback
}

\author{
$X u$ Guangwen, $\mathrm{Li} \mathrm{Xi}^{*}$, and Yao Ze \\ Electric Power Research Institute of Guangdong Power Grid Co., Ltd., Guangzhou 510080, Guangdong, China
}

\begin{abstract}
To solve the damping problem of water hammer wave in the modeling method of water diversion system of hydropower station, this paper introduces the feedback regulation technology from head to flow, that is: A fixed water head is taken out for flow feedback, and the following conclusions are obtained through modeling and simulation. Adjusting the feedback coefficient $\mathrm{F}$ of the water head to the flow rate can change the damping characteristic of the system, which can simulate the attenuation process of the water shock wave in the true water diversion pipeline. Even if a small feedback coefficient is introduced, the damping effect of the system is very obvious, but it has little effect on the amplitude of the first water shock wave after the transition process. Therefore, it is feasible and reasonable to introduce water head to flow rate feedback coefficient $\mathrm{F}$ in hydraulic turbine diversion system.
\end{abstract}

\section{Introduction}

In order to make rational use of water energy and take account of the feasibility and economy of hydropower development, the layout and structure of diversion system of hydropower stations are usually varied. Generally speaking, it is mainly composed of upstream and downstream reservoirs, pressure conduit, turbine runner and draft tube [1]. A simple single pipe and single machine diversion system is simply shown in Figure 1.

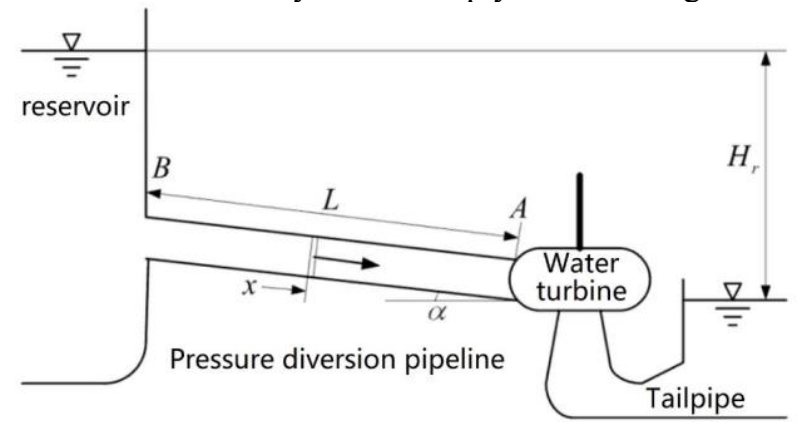

Fig. 1. Water diversion system of a typical hydropower station.

For the modeling or calculation method of the water diversion system, the linear transfer function description method [2] can be used when the length of the pipe is less than $600 \sim 800$ meters. If the diversion pipe is longer, the effect of the elastic water hammer should be considered [3-5], and the characteristic line method is usually used. It is pointed out that when the flow of water in the diversion pipeline is rapidly cut off, the pressure wave in the pipeline is very slow, which is not consistent with the actual situation. In order to overcome these defects, in the nonlinear simulation calculation of the hydraulic turbine governing system, this paper improves the characteristic line method, and introduces the water head to the flow feedback to the modeling of the water diversion system of the hydropower station, and the simulation is carried out. The results are reasonable and credible.

\section{Basic characteristic line method and calculation example}

\subsection{An example of the basic characteristic line method}

The basic characteristics of line method for modeling as shown in Figure 2, figure symbol "| - |" absolute value calculation

\footnotetext{
*Corresponding author: lilixi5217@163.com
} 


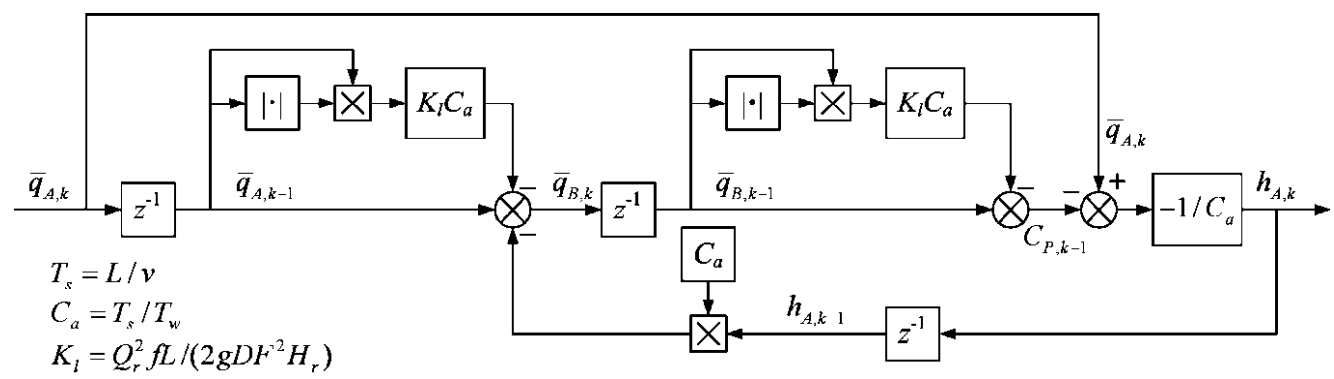

Fig. 2. The basic characteristic line block diagram for single section calculation of simple water diversion pipeline.

\subsection{Calculation example}

The parameters of a hydraulic turbine guide system are as follows: the length of the equivalent water diversion pipe: $\mathrm{L}=200$ meters; water hammer wave speed: $\mathrm{V}=1000$ $\mathrm{M} / \mathrm{sec}$; calculation step length: $\mathrm{T}_{\mathrm{s}}=\mathrm{L} / \mathrm{v}=0.2$; the speed constant of water flow: $\mathrm{T}_{\mathrm{w}}=2$ seconds; the water head loss coefficient is $\mathrm{K}_{1}=0.02$ and $\mathrm{K}_{1}=0.1$, respectively, assuming that the flow of the turbine is proportional to the opening of the relay, and is linear from full open to full or full off in 5 seconds. The initial conditions of all the variables were 0 . The simulation results are shown in Figure 3 and Figure 4.

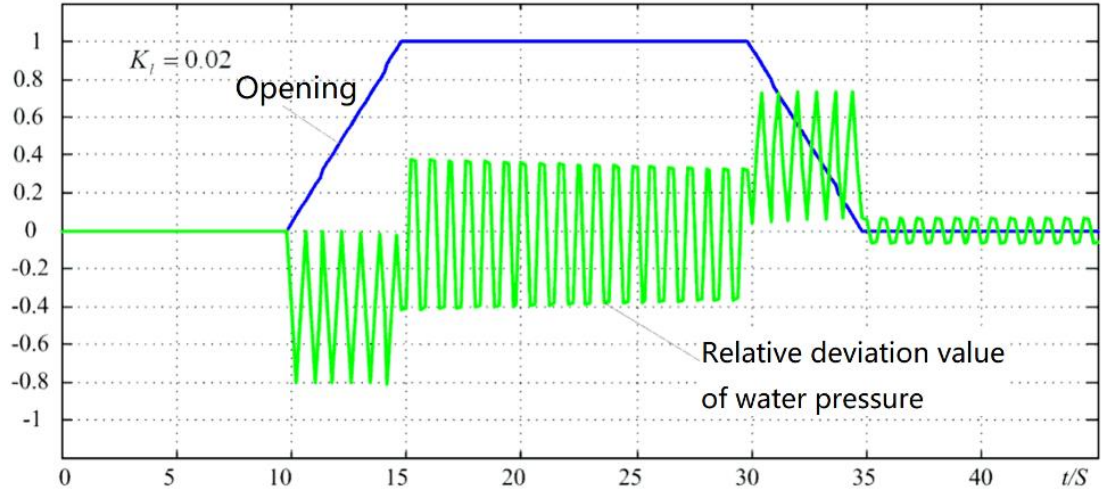

Fig. 3. Water pressure deviation response process of basic characteristic line method at $K_{l}=0.02$.

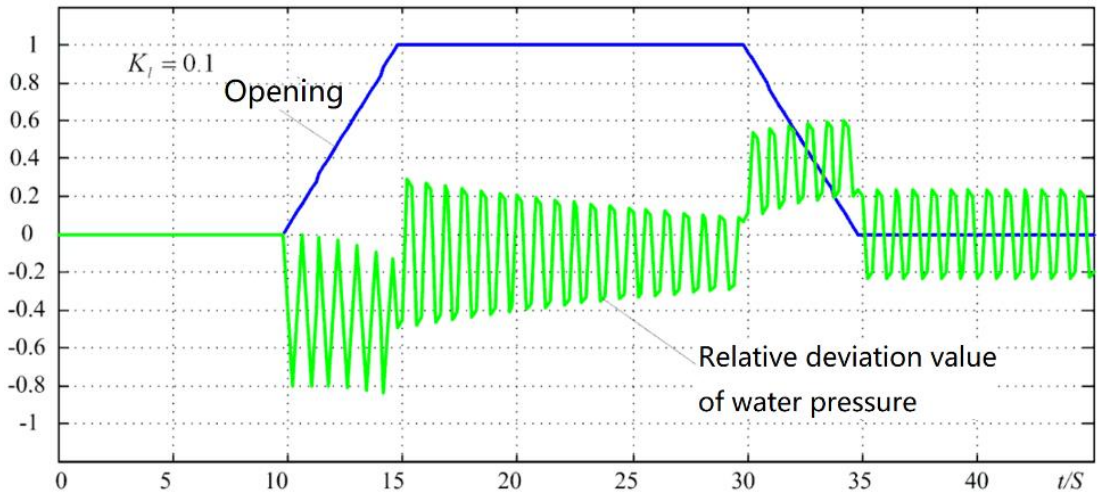

Fig. 4. Water pressure deviation response process of basic characteristic line method at $K_{l}=0.1$.

As can be seen from Figure 3 and Figure 4, the basic characteristic line method discussed can show the properties of elastic water hammer, but the elastic water strike wave attenuates slowly and the damping is smaller, especially after the water flow is cut off. This situation is usually not in conformity with the reality. It is generally considered that the attenuation of elastic water hammer wave is related to the head loss coefficient $\mathrm{Kl}$, and the larger the head loss coefficient is, the faster the attenuation is. However, the two diagram shows that the underdamped condition has a certain relationship with the water head loss coefficient, but the correlation is not significant. Even if the $K_{1}$ is increased from 0.02 to 0.1 , the damping condition does not improve when the flow is cut off. This further verifies the damping problem of the head wave in the basic characteristic line method proposed at the beginning of this section, especially after the sudden flow cut-off.

\section{Improved characteristic line method and its calculation example}

\subsection{A linear discrete model with water head to flow feedback}

Since the damping of the head wave has little relation with the loss coefficient of the head, the water head loss item in the characteristic line algorithm can be removed and the linear model based on the characteristic line method is established. Moreover, based on the linear model, it is easy to control damping and transform the sampling period. On the basic characteristic line block 
diagram of single section calculation of water diversion pipeline, after removing the head loss item and introducing the feedback from water head to flow, the algorithm block diagram is changed to figure 5 .

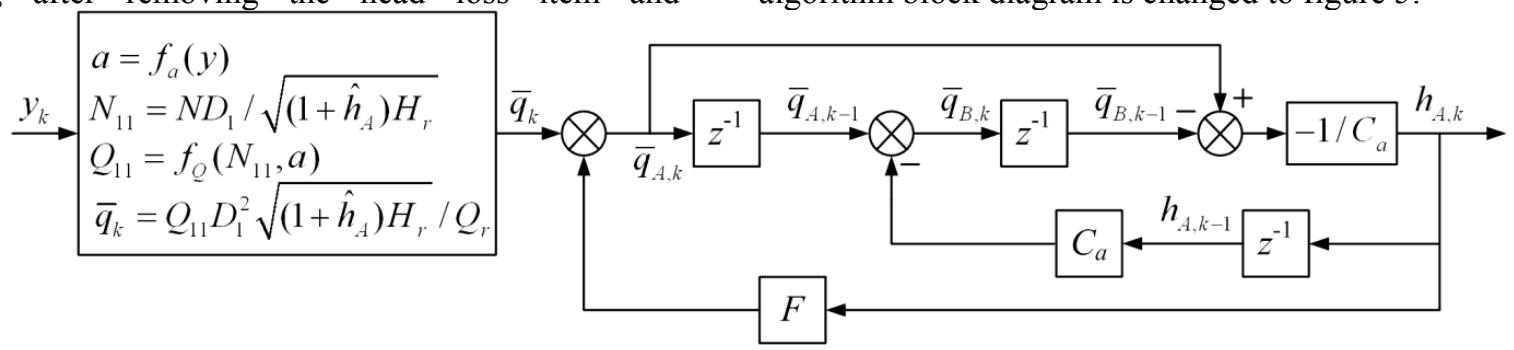

Fig. 5. Water pressure deviation response process of basic characteristic line method at $K_{l}=0.1$.

In Figure $5, F$ is the feedback coefficient of additional water head to flow, $\bar{q}_{k}$ is the relative flow volume of turbine and $\hat{h}_{A}$ is the estimated value of water head increment during iteration. Here we only discuss the linear model from the $\bar{q}_{k}$ to $h_{A, k}$.First, we write the discrete form of the system ( $Z$ transfer function) equation according to the 5 column of block diagram.

$$
\begin{gathered}
Q_{B}(z)=Q_{A}(z) z^{-1}-C_{a} H_{A}(z) z^{-1} \\
H_{A}(z)=-\frac{1}{C_{a}}\left(Q_{A}(z)-Q_{B}(z) z^{-1}\right) \\
Q_{A}(z)=F H_{A}(z)+Q(z)
\end{gathered}
$$

For the sake of derivation, the $\mathrm{Z}$ transformation of all variables is expressed by capitalized letters of variables. Take (3) into (2) and (1).

$$
\begin{gathered}
Q_{B}=\left(F H_{A}+Q\right) z^{-1}-C_{a} H_{A} z^{-1} \\
H_{A}=-\frac{1}{C_{a}}\left(F H_{A}+Q-Q_{B} z^{-1}\right)
\end{gathered}
$$

Then bring (5) into (4)

$$
H_{A}=\frac{1}{C_{a}}\left(-F H_{A}-Q+F H_{A} z^{-2}+Q z^{-2}-C_{a} H_{A} z^{-2}\right)
$$

After arranging

$$
\left(F+C_{a}-\left(F-C_{a}\right) Z^{-2}\right) H_{A}=Q\left(-1+Z^{-2}\right)
$$

The corresponding $\mathrm{Z}$ transfer function is

$$
G(z)=\frac{H_{A}(z)}{Q(z)}=\frac{-1+z^{-2}}{\left(F+C_{a}-\left(F-C_{a}\right) z^{-2}\right)}
$$

If $\mathrm{F}=0$, it is equivalent to the case of no additional water head to the feedback coefficient of flow, which is equivalent to the basic characteristic algorithm model without considering head loss.

$$
G(z)=\frac{H_{A}(z)}{Q(z)}=\frac{H_{A}(z)}{Q_{A}(z)}=\frac{1}{C_{a}} \frac{-1+z^{-2}}{1+z^{-2}}
$$

\subsection{Calculation example}

In order to verify the feasibility of the proposed method, the improved characteristic line method is simulated by combining 2.2 sections of turbine intake system parameters.

The feedback coefficients of the head to flow are taken as $\mathrm{F}=0, \mathrm{~F}=0.001, \mathrm{~F}=0.01$ and $\mathrm{F}=0.1$, and assume that the flow of the turbine is proportional to the opening of the relay and linearly from full open to full or full off in 5 seconds. The initial conditions of all the variables were 0 . The simulation results are shown in figure $6 \sim 9$.

When the feedback coefficient is $F=0$, the system is unstable and its time domain response is an obvious equal amplitude oscillation. With the increase of the feedback coefficient, the damping of the response output increases and the attenuation accelerates. When the feedback coefficient is $F=0.1$, the system has the characteristic of over damping and has no oscillation characteristics; the adjustment of the feedback coefficient $\mathrm{F}$ can change the damping characteristic of the system and thus can change the damping characteristic of the system. The attenuation process of water hammer wave in real water diversion pipeline is simulated. Even if a small feedback coefficient is introduced, it is very obvious to increase the damping of the system, but it has little effect on the amplitude of the first water shock wave after the transition process. Therefore, it is feasible and reasonable to introduce water head to flow rate feedback coefficient $F$ in hydraulic turbine diversion system.

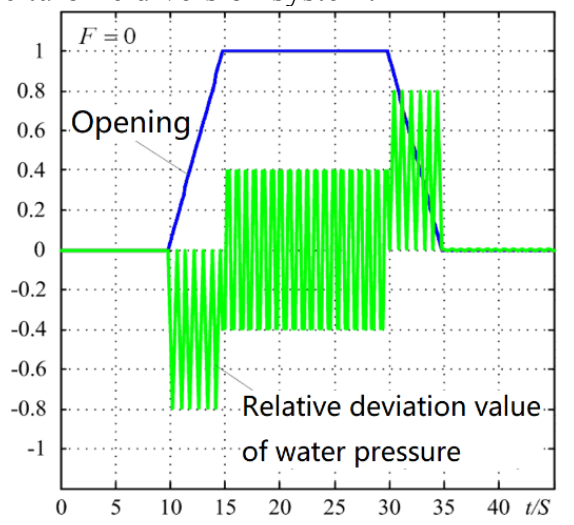

Fig. 6. Response of the system to feedback, $F=0$.

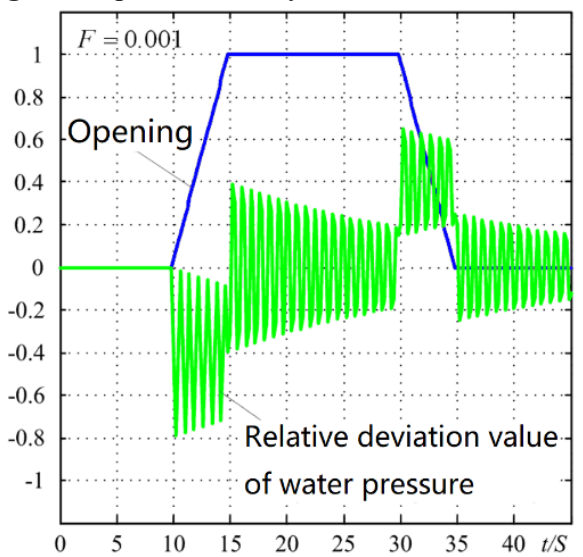

Fig. 7. Response of the system to feedback, $F=0.001$. 


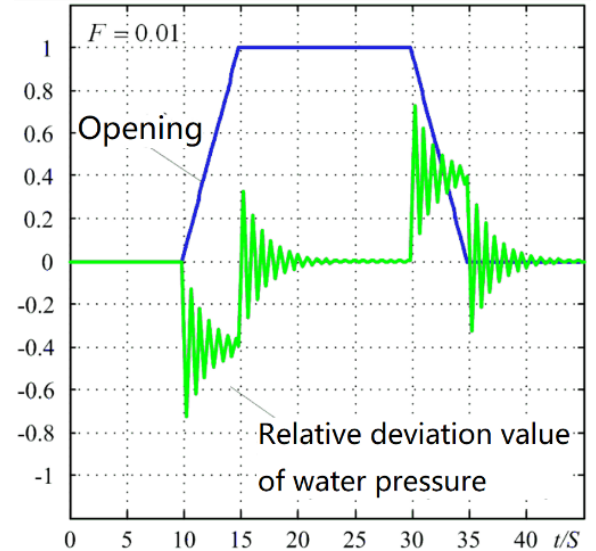

Fig. 8. Response of the system to feedback, $F=0.01$.

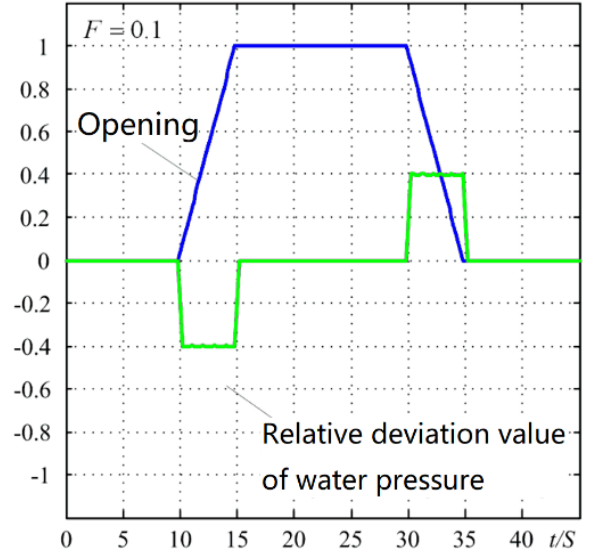

Fig. 9. Response of the system to feedback, $F=0.1$.

\section{Conclusion}

The modeling method of water diversion system of hydropower station, that is, the characteristic line method, even to the solution of a pair of nonlinear hyperbolic partial differential equations, is to solve the damping problem of water shock wave. In this paper, the feedback regulation technology of water head to flow is introduced in this paper, and a fixed head of water head is taken out to feed back the flow. The results show that the reasonable feedback coefficient is helpful to the damping of water shock wave. The modified model is reasonable. The adjustment of the feedback coefficient $F$ can change the damping characteristic of the system, which can simulate the attenuation process of water shock wave in the true water diversion pipeline. Even if a small feedback coefficient is introduced, the damping effect of the system is very obvious, but the amplitude of the first water shock wave after the transition process has little effect. Therefore, it is feasible and reasonable to introduce water head to flow rate feedback coefficient $F$ in hydraulic turbine diversion system.

\section{Acknowledgments}

The research work was supported by Science and technology project of Guangdong Power Grid Co., Ltd. (GDKJQQ20152035).

\section{References}

1. Liu H, Lai C, Yuan Q, et al. Study on Optimization Plan of Water Diversion System with High-head and Small Flow Hydropower Station[J]. South-to-North Water Transfers and Water Science \& Technology. 2010 , $8(1672-$ 1683(2010)8:5<149:GSTXLL>2.0.TX;2-S5): 149 152.

2. Cai D, Zhao J. ANALYSIS OF AXISYMMETRIC FORWARD/BACKWARD EXTRUSION BY THE CHARACTERISTIC LINE METHOD.[J]. Ku Ti Li Hsueh Hsueh Pao/Acta Mechanica Solida Sinica. 1987(2): 116-126.

3. Chang Y, Xi Y, Fan Y. Analysis on transient flow of unpressurized water diversion system for Manweilai Hydropower Station in Cameroun[J]. Water Resources and Hydropower Engineering. 2017, 48(1000-0860(2017)48:10<31:KMLMWL>2.0.TX; 2-O10): 31-36.

4. Guo W, Yang J, Shan X. Pipeline system's hydraulic design of multi-water-source diversion-type hydropower station[J]. Engineering Journal of Wuhan University. Engineering Edition. 2013, 46(1671-8844(2013)46:1<73:DSYYSS>2.0.TX;2\#1): 73-77, 83.

5. Abi E, Zheng Y, Feng X, et al. Modification and development of characteristic line method of tunnel[J]. Yanshilixue $\mathrm{Yu}$ Gongcheng Xuebao/Chinese Journal of Rock Mechanics and Engineering. 2015, 34: 3067-3073. 